\title{
UTILIZING KNOWLEDGE OF PHYSICS IN SAFETY ENGINEERING
}

Any technician working in safety engineering needs certain knowledge of physics. This knowledge includes physical quantities and units of measurement, physical properties of substances, various formulae and equations, etc. It is also very important to possess knowledge enabling general - and often highly complex - processes to be simplified. This paper discusses two such possibilities for simplification.

Key words: physical field, heat transfer

\section{Introduction}

When solving various practical problems of safety engineering, general solutions may be highly complex [5]. It is therefore of key importance whether the solution may be simplified, and if so under what conditions, as well as whether certain processes may be disregarded. This paper presents and discusses two such possibilities for simplification.

\section{Point sources and non-point sources of physical fields}

The solutions to a range of physical and technical problems involve various physical fields (gravitation, electrical, thermal, acoustic, etc.). In most cases it is the intensity and potential of these fields that are used in calculations.

When carrying out such calculations it is important whether the source of the physical field is a point source (in which case the calculation is simple) or a non-point source (which complicates the calculation).

A point source of a physical field is a source whose dimensions and shape can be disregarded when solving the given problem. The dimensions and shape of a non-point source cannot be disregarded. Non-point sources of physical fields include linear sources (e.g. wires), surface sources or general sources (bodies).

The condition for simplification is determined depending on the accuracy with which the calculation is to be carried out. It is usually sufficient if the difference between the quantity calculated for a field produced by a point source $\left(x_{b}\right)$ and the quantity calculated generally for a non-point source of a field $(x)$ is no larger than one percent, i.e.

$$
x_{b}=1.01 x
$$

The following examples show how it is possible to work with the concepts of point sources and non-point sources of physical fields.

\subsection{Electrical fields}

\section{Intensity of electrical fields}

The intensity of an electrical field produced by a point electrical charge in a vacuum is calculated by the equation

$$
E=\frac{1}{4 \pi \varepsilon_{0}} \frac{Q}{r^{2}}\left(N C^{-1}, V m^{-1}\right)
$$

where

$\varepsilon_{0}$ - permittivity of vacuum

$Q$ - charge (C)

$r \quad$ - distance from charge $(\mathrm{m})$

However, this equation is only accurate for point charges or charged homogeneous balls (the distance is measured from the centre of the ball).

If the charged body is not a point source, the situation is somewhat more complicated, and the intensity of the electrical field must be calculated according to the equation

$$
E=\frac{1}{4 \pi \varepsilon_{0}} \int \frac{d Q}{r^{2}}
$$

where

$d Q$ - element of electrical charge.

\footnotetext{
* Jiri Svec ${ }^{\mathbf{1}}$, Radomir Scurek ${ }^{\mathbf{1}}$, Pavel Svec ${ }^{\mathbf{2}}$,

${ }^{1}$ Department of Safety Management, Faculty of Safety Engineering, VSB-Technical University of Ostrava, Czech Republic, E-mail: jiri.svec@vsb.cz.

${ }^{2}$ Department of Automation and Computing in Metallurgy, Faculty of Metallurgy and Materials Engineering, VSB-Technical University of Ostrava, Czech Republic.
} 


\section{Electrical field of a charged ring}

A thin wire is formed into a ring with radius $a$, and the total electrical charge of the ring is $Q$. The intensity of the electrical field is determined at point $P$ on the axis of the ring at distance $b$ from the centre of the ring (Fig. 1).

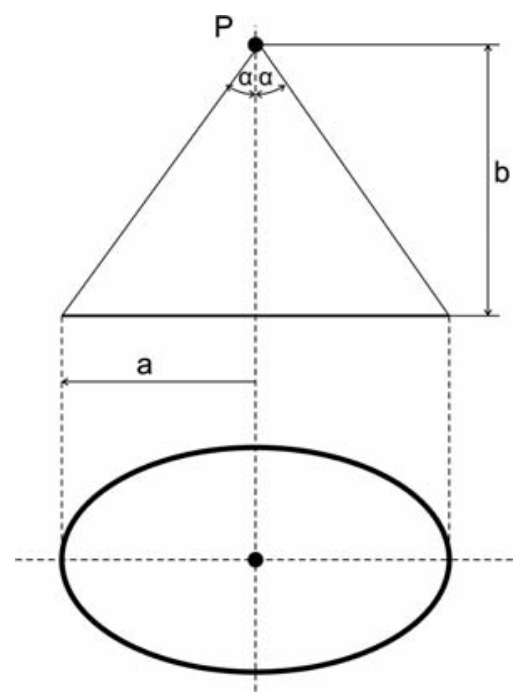

Fig. 1 Calculation of the intensity of the electrical field of a charged ring

Using equation (3), it is possible to derive for the given intensity the equation [1] and

$$
E=\frac{Q}{4 \pi \varepsilon_{0}} \frac{b}{\left(a^{2}+b^{2}\right)^{\frac{3}{2}}}
$$

The quantities are marked as in Fig. 1.

If distance $b$ is much larger than the radius of the ring $a-$ i.e. $b>>a-$ then equation (4) can be simplified to

$$
E=\frac{1}{4 \pi \varepsilon_{0}} \frac{Q}{b^{2}}
$$

which corresponds with the equation for the intensity of a point charge located in the centre of the ring.

The condition for simplification for a $1 \%$ difference in intensity calculated generally (equation 4 ) and in a simplified manner (equation 5) can be determined from the following equation (according to equation 1):

$$
\frac{1}{4 \pi \varepsilon_{0}} \frac{Q}{b^{2}}=1.01 \frac{Q}{4 \pi \varepsilon_{0}} \frac{1}{\left(a^{2}+b^{2}\right)^{\frac{3}{2}}}
$$

The solution of this equation gives us the result

$$
b=11.9 a \doteq 12 a
$$

Thus, if the distance from the centre of the ring is over 12 times more than the radius of the ring, the calculation of the intensity of the electrical field can be carried out using the equation for the calculation of the intensity of the electrical field of a point charge located in the centre of the ring.

\subsection{Light fields}

Illumination with a surface source

The illumination of point $P$, which lies on the axis of a circular disc with radius $a$ and luminance $L$ at distance $g$ from the centre of the disc (Fig. 2), is calculated according to equation [2]

$$
E=\frac{L S}{a^{2}+g^{2}}
$$

where $S=\pi a^{2}$

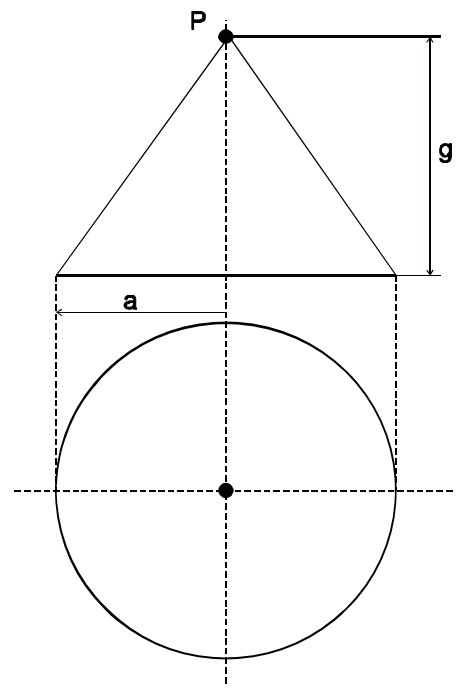

Fig. 2 Calculation of illumination from a surface source

Due to the sufficient distance of point $P$ from the disc, we can consider the disc as a point source of light, and calculate the illumination on the basis of the equation

$$
E=\frac{L S}{g^{2}}
$$

The condition for simplification for a $1 \%$ difference in the the quantity of illumination calculated generally (7) and in a simplified manner (8) can be determined from the following equation (according to equation 1):

$$
\frac{L S}{g^{2}}=1.01 \frac{L S}{a^{2}+g^{2}}
$$

The solution of this equation gives us the result

$$
g=10 a
$$


Thus, if the distance $\mathrm{g}$ from the centre of the ring is 10 times larger than the disc radius a, the disc can be considered a point source of light.

\section{Heat transfer}

Heat transfer plays an important (though often neglected) role in the solution of thermokinetic problems [6]. It is a process which occurs on the boundary of gas or liquid and a solid substance, i.e. on the boundary where convection changes to conduction (or vice versa).

At this boundary there is a sharp jump in temperature. In the liquid (or gas) the temperature is approximately constant except for a very thin layer adjacent to the wall, in which the temperature drops significantly, so the wall has a lower temperature than the temperature of the liquid. This occurs in cases where the heat is transferred from the liquid to the wall. In the opposite case, when the heat is transferred from the wall to the liquid, the wall has a higher temperature than the liquid.

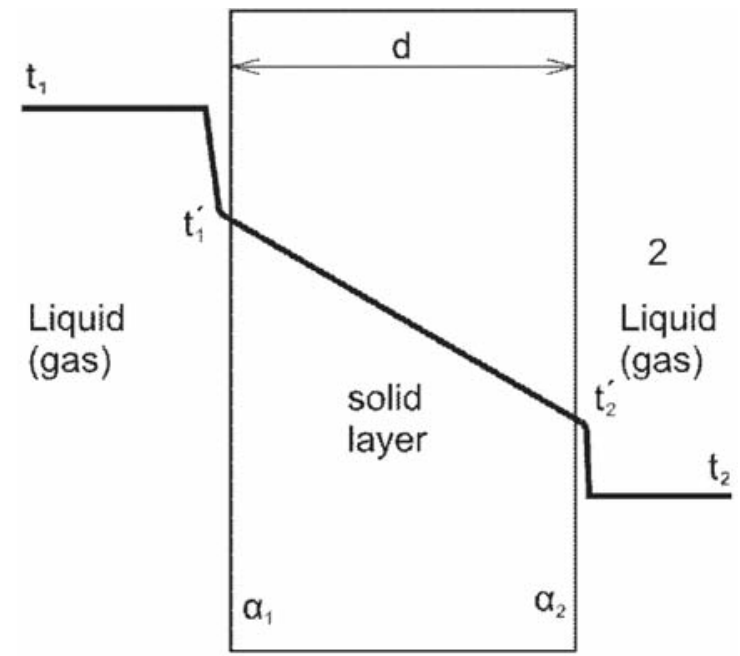

Fig. 3 Temperature change during heat transfer from liquid to liquid via a solid layer

If the liquid (Fig. 3) has temperature $t_{1}$ and the surface of the solid wall has temperature $t^{\prime}{ }_{1}$, then the heat transferred during time $\tau$ through surface $S$ from the liquid to the wall is given by Newton's equation

$$
Q=\alpha S \tau\left(t_{1}-t_{1}^{\prime}\right)
$$

The proportionality constant $\alpha$ is known as the heat transfer coefficient $\left(\mathrm{Wm}^{-2} \mathrm{~K}^{-1}\right)$.

In practice, we usually determine the penetration of heat from one liquid (gas) to another liquid (gas) via a flat wall. In a stationary state, it is easy to derive the equation
$Q=k S \tau\left(t_{1}-t_{2}\right)$

The quantities are marked as on Fig. 3.

Constant $k$ is known as the heat penetration coefficient $\left(\mathrm{Wm}^{-2} \mathrm{~K}^{-1}\right)$.

$$
\frac{1}{k}=\frac{1}{a_{1}}+\frac{d}{\lambda}+\frac{1}{a_{1}}
$$

where

$\alpha_{1}, \alpha_{2}$ - heat transfer coefficient from liquid 1 to the wall or from the wall to liquid $2\left(\mathrm{Wm}^{-2} \mathrm{~K}^{-1}\right)$

$d$ - wall thickness (m)

$\lambda$ - thermal conductivity coefficient of the wall material $\left(\mathrm{Wm}^{-1} \mathrm{~K}^{-1}\right)$

At first sight it may appear that heat transfer is not a particularly complex problem. However, the reality is different. Unlike the thermal conductivity coefficient, which is a material constant, the heat transfer coefficient is not a material constant. It depends on a range of parameters, e.g. density, viscosity, thermal conductivity of the medium, convection speed, geometric and shape parameters, etc. It can reach highly divergent values depending on circumstances (Table 1):

Approximate range of values of the heat

Table 1 transfer coefficient [3]

\begin{tabular}{|c|c|}
\hline Coefficient without phase change & $\alpha\left(\mathrm{W} \mathrm{m}^{-2} \mathrm{~K}^{-1}\right)$ \\
\hline Natural convection gases & $3-20$ \\
\hline water & $100-600$ \\
\hline Forced convection gases & $10-500$ \\
\hline water & $500-10000$ \\
\hline highly viscous liquids & $50-500$ \\
\hline Coefficient with phase change & $1000-20000$ \\
\hline liquid boiling & $1000-100000$ \\
\hline vapour condensation &
\end{tabular}

For this reason, equations (10), (11) and (12) are simple only in formal terms. Determining the heat transfer coefficient (and thus also the heat penetration coefficient) is a complex problem, and exceeds the scope of this paper.

As an illustration, we can calculate the heat flow (the heat flowing through a surface of $1 \mathrm{~m}^{2}$ in $1 \mathrm{~s}$ ) for the following conditions (markings according to Fig. 3).

1. $t_{1}=5{ }^{\circ} \mathrm{C}, \alpha_{1}=7.5 \mathrm{~W} \mathrm{~m}^{-2} \mathrm{~K}^{-1}$, copper layer, $\mathrm{d}=1 \mathrm{~cm}$, $\lambda=372 \mathrm{~W} \mathrm{~m}^{-1} \mathrm{~K}^{-1}, \alpha_{2}=50 \mathrm{~W} \mathrm{~m}^{-2} \mathrm{~K}^{-1}, t_{2}=45^{\circ} \mathrm{C}$. 
2. $t_{1}=5{ }^{\circ} \mathrm{C}, \alpha_{1}=7.5 \mathrm{~W} \mathrm{~m}^{-2} \mathrm{~K}^{-1}$, polystyrene layer, $\mathrm{d}=1 \mathrm{~cm}$, $\lambda=0.06 \mathrm{~W} \mathrm{~m}^{-1} \mathrm{~K}^{-1}, \alpha_{2}=50 \mathrm{~W} \mathrm{~m}^{-2} \mathrm{~K}^{-1}, t_{2}=45^{\circ} \mathrm{C}$.

In the first case (a) we calculate the heat flow including heat transfer; in the second case (b) the heat transfer on both sides of the solid layer is disregarded.

The results can be summarized as follows:

$$
\begin{aligned}
& 1 \mathrm{a} \text {............ } q=267 \mathrm{~W} \mathrm{~m}^{-2} \quad 1 \mathrm{~b} \ldots \ldots \ldots \ldots . . . . q=1.48 \mathrm{MW} \mathrm{m}^{-2} \\
& 2 \mathrm{a} \ldots \ldots \ldots \ldots . . . . q=125 \mathrm{~W} \mathrm{~m}^{-2} \quad 2 \mathrm{~b} \ldots \ldots \ldots \ldots . . . q=240 \mathrm{~W} \mathrm{~m}^{-2}
\end{aligned}
$$

It is evident that heat transfer plays a major role in the solution of thermokinetic problems - even those problems which seem simple. Analysis [4] shows that the influence of heat transfer is particularly significant in the case of highly conductive solids, growing as the solid layer becomes thinner. If we intend to disregard this phenomenon, there must be very good reasons for doing so. Otherwise, calculations may be burdened with significant error.

\section{Conclusion}

When solving complex practical problems, we search for opportunities to simplify. However, it is essential to consider in which conditions such simplifications may be made, and what effect this will have on the resulting solution of the problem.

This contribution was prepared in the framework of dealing with the grant project of the Ministry of the Interior of the Czech Republic, Security Research Programme, under the No. MV0400511, "Influence of Terrorist Attack on Selected Industrial Technologies with a Dust Explosion Hazard”.

\section{References}

[1] FUKA, J., HAVELKA, B.: Electricity and Magnetism (in Czech), SNTL, Praha 1965.

[2] FUKA, J., HAVELKA, B.: Optics (in Czech), SPN, Praha 1961.

[3] BLAHOZ, V., KADLEC, Z.: Basis of Heat Sharing (in Czech), 2. vydani, Ostrava : SPBI v Ostrave, 2000, ISBN 80-902001-1-7.

[4] SVEC, J., SVEC, P.: Heat Transfer (in Czech), In. Sborník vedeckych praci VSB-TU Ostrava, Rada bezpecnostni inzenyrstvi, Vol. V, No. 1, Ostrava 2010, pp. 101-108, ISBN 978-80-248-2317-1.

[5] KLOUDA, K., BRADKA, S.: Also the $4^{\text {th }}$ Dimension of the Town/its Underground Structures - has its Risks, In: Communications - Scientific Letters of the University of Zilina, Vol. 10, 2008, p. 5-9, ISSN 1335-4205.

[6] STROCH, L.: Explosion Preventive in the Present Conditions of the Production, In: Communications - Scientific Letter of the University of Zilina, Vol. 10, 2008, p. 60-64, ISSN 1335-4205. 